\title{
The Causes, Effects and Control of Bronchial Secretions
}

\author{
F. A. MEINTJES, F.R.C.S.Ed.
}

(Department of Thoracic Surgery, Pretoria Hospital)

\begin{abstract}
With the exception of the skin, which has cornified and in fact dying and continually desquamating protective superficial layer, all lining or covering membranes, such as for example, the bronchial mucosa or the pleura require a moist environment for their survival and satisfactory function. Such an environment is achieved to a greater or lesser extent by the secretion of mucus or serous fluid onto the surfaces of such membranes, and thus also into the lumens of organs or tracts lined by them. In the specific instance of the tracheo-bronchial tree an important and highly specialised function of the mucous membrane, viz. the ciliary action, is amongst those dependent on the presence of a microscopic film of mucus on the surface. The fine hair-like cilia projecting from the cells wave constantly back and forth in such a manner that this layer of mucus, along with any foreign particles or bacteria which are present in it, is passed steadily upwards and eventually expelled into the laryngo-pharynx. Should any inflammatory reaction occur in the bronchi, the secretion of mucus will increase, leading perhaps to accumulation thereof, and this will necessitate the bringing into action of a further expulsive mechanism, viz. that of coughing.
\end{abstract}

\section{THE COUGH MECHANISM}

Cough may be defined as an initial deep inspiration followed by the sudden explosive release of air from the lungs due to the forcible contraction of the abdominal muscles while the chest wall is immobilised and the glottis (vocal cords) initially closed (leading to a significant pressure build-up) and then suddenly opened. Thus secretions or foreign bodies tend to be eliminated by a rapidly-moving column of air BEHIND them. The nervous control of the act is dependent firstly on normal sensation in the bronchi, trachea, larynx and even the pharynx (particularly the pyriform fossa), which are the various sites at which an irritant stimulus or pathological process giving rise to cough may occur. Secondly, the nervous pathways must be intact- that is, sensory nerves, cerebral centres and motor nerves. And finally, for the actual performance of the act of coughing, muscle power must be adequate.

Logically now, the possible CAUSES OF INTERFERENCE with the cough mechanism may be enumerated as follows:

1. If the patient is unwilling or unable for any reason to make the effort, e.g. lack of pre-operative training, or the presence of upper abdominal or thoracic pain, poor mental attitude, etc.

2. If the sputum is unduly tenacious.

3. If the respiratory (ventilatory) function is impaired, e.g. operative or accidental chest trauma, bronchospasm, emphysema, atalectasis, air or fluid in the pleural cavity, chest deformity (kypho-scoliosis, funnel-chest, pigeon-breast) or muscular weakness.

4. If bronchial sensation is impaired as after the use in the trachea and bronchi of a local anaesthetic spray (frequently employed as an adjunct to general anaesthesia, and occasionally to permit of bronchoscopy without general anaesthesia).

5. If movement of the vocal cords is impaired by paralysis, or if their effect is eliminated by the existence of a tracheostomy opening.
6. If the relevant nervous pathways are interrupted, as in cases of deep unconsiousness, high spinal cord injuries or diseases, poliomyelitis, etc.

Such impairment of the cough reflex, or alternatively the pathological over-production of secretions, will lead to ACCUMULATION OF MATERIAL in the tracheobronchial tree, with the following harmful effects.

1. Stasis leads to infection, and as infection brings about increased secretory activity, a vicious circle is established and eventually frank purulent changes occur. Also the ciliary action is interfered with. Infected secretion may then, just as does infected foreign material, lead to the development of bronchopneumonia or lung abscesses.

2. Bronchial obstruction is followed by the absorption of the air in the lung distal to the obstruction, with resultant atalectasis (collapse), and furthermore the absence of air BEHIND such an obstruction renders coughing less effective.

3. Interference with ventilation which in severe cases causes dyspnoea and cyanosis. Should there already be present one of the abnormalities (already mentioned) which themselves impair ventilation, even a minor degree of bronchial obstruction may have dire effects.

However, much can be done by the doctor and by the physiotherapist to assist the patient by ARTIFICIAL MEANS in the elimination of his bronchial secretions.

1. Controlled breathing exercises and postural drainage. By means of accurate positioning of the patient according to specific anatomical considerations, gravity is invoked to facilitate the drainage of individual lobar and segmental bronchi.

2. Pain control, by means of frequent administration of adequate although not heavy doses of pain-killing drugs, the object being to allevaite chest or abdominal pain without sedating the patient to such an extent that he is unable to co-operate with the physiotherapist, or that his respiration is depressed. (The assistance of the physiotherapist in assessing a patient's needs in this respect is much sought after by medical officers in charge of thoracic surgical cases.)

3. Mucus may be rendered less viscid through lowering its surface tension by means of inhalation of moisture, or of more specific vapours which have this effect or by the administration systematically of mucolytic enzymes.

4. Antispasmodic drugs, for the relief of bronchospasm.

5. Treatment of the associate chest abnormality, e.g. fixation of unstable ribs or sternum resulting from injury, or drainage of fluid or air from the pleural cavity.

6. It is occasionally necessary to actively remove secretions from the bronchial tree by means of suction, either through a bronchoscope, necessitating general anaesthesia or by catheter through a tracheostomy which may already have been constructed, or which may be performed specifically for this purpose. 
THE PRODUCTIVE COUGH AND

COMMON COMPONENTS OF SPUTUM

The type and quantity of sputum produced by patients with pulmonary or cardiac disease are significant, and variations thereof may be logically explained on the basis of the underlying causal mechanism.

1. Simple Over-production of Normal Mucus, e.g. by mechanical irritation as by an endo-tracheal tube when pre-medication for anaesthesia has been inadequate, or by chemical irritation (pungent gases) or as a result of an allergic reaction (c.f. the excessive watery nasal discharge associated with hay-fever.

2. Pulmonary Oedema-the pouring-out of fluid from the pulmonary capillaries into the alveoli (i.e. through the membrane across which only gaseous exchanges between the blood and the atmosphere take place normally), due to increased hydrostatic pressure in the capillaries. This may result from increased blood-flow into them, as occurs for example after the repair of certain congenital heart lesions (pulmonary stenosis) or after over-transfusion or alternatively it may be due to obstruction to the pulmonary venous return to the heart, as in the case of stenosis of the mitral valve. The sputum is typically frothy, due to intimate mixing with air in the alveoli, and may also be blood-stained if the distended capillaries should rupture. Because simple excessive hydrostatic pressure is the cause, a postural factor operates, that is the lower the trunk of the patient in relation to the rest of his body, the higher the pressure in his pulmonary capilliary bed, and therefore the greater the tendency towards pulmonary oedema. Characteristically patients suffering from this condition are acutely short of breath, cyanosed, produce large quantities of the sputum described with little effort, and are unable to lie down without much aggravation of complaints.

3. Haemoptysis. Blood in the sputum-may be due to rupture of vessels by distension, erosion, or injury.

The distension of the pulmonary capillary bed associated with pulmonary oedema has already been mentioned. But more frequently the vessels of the bronchial mucosa or of the lung become congested as a result of inflammation as in the case of bronchitis, pneumonia, or tuberculosis.

Thirdly, in compensating for obstruction to bloodflow into the lung via the pulmonary artery, the bronchial arteries (arising from the systemic arterial tree as opposed to the pulmonary circulation) establish a collateral flow, and in so doing distend and frequently rupture. The most common instance is that of the late stage of mitral stenosis, which is associated with pulmonary hypertension.

Erosion of bronchial mucous membrane results from tumour-growth, most significantly in the case malignant infiltrative lesions such as carcinoma of the bronchus itself (loosely known also as carcinoma "of the lung"), or in association with malignancy of adjoining structures (e.g. the oesophagus) by direct extension. However, benign tumours, may also ulcerate and present with haemoptysis, as is frequently the case with a bronchial adenoma.

Injury of the lung or bronchi may be due to penetration as by stabbing, or by displacement of fractured ribs, or to external compression, particularly as a result of the all too common "steering-wheel" injury.

Blood may also be coughed out after having been inhaled after haemorrhage from the upper respiratory passages_-epistaxis, or after tonsillectomy. And lastly massive haemorrhage into the trachea and bronchi may result from rupture of an aortic aneurysm, and is is usually followed by death.

\section{Unusual Constituents of Sputum:}

Rupture of an hydatid cyst (that stage of the lifecycle of the parasite taenia echinococcus which occurs in man) releases clear watery fluid, and dissemination of the infestation may be associated with the coughing out of these contents.

Similarly a dermoid (developmental) cyst may drain into a bronchus, and the patient thus produce sputum containing various skin-derivatives and secretions, such as sebaceous material or hair.

Pulmonary Mucoviscoidosis. This is a condition occurring in new-born and very young children, in association with fibrosis of the pancreas (and disturbance of its enzymatic function). Secretions become extremely tenacious and cause interference with pulmonary ventilation due to bronchial obstruction, and in the event of survival these circumstances lead to the development of a cystic type of bronchiectasis.

\section{Conditions Giving Rise to Purulent Sputum:}

\section{Bronchitis.}

Bronchiectasis.

Lung Abscess.

Empyema with a broncho-pleural fistula.

Purulent sputum varies greatly in character and amount and the possible cause thereof and the effectiveness of therapy may be judged accordingly. Thus a patient producing much thick offensive sputum (e.g. from a lung abscess) will on intensive postural drainage (with or without the use of antibiotics in addition) respond by producing thinner, lightercoloured material, the volume of which will eventually also start decreasing. Associated with the improvement in the sputum there will also be an improvement in the patient's general condition.

Offensiveness is related to two factors-the amount of tissue destruction (putrefaction) and the type of causative organism. "Gangrene of the lung" is due to a fulminating infection, so severe that the tissue dies and sloughs, portions of this slough possibly even appearing in the sputum, which classically is pungently foul-smelling. However, this is a rare condition today.

More commonly offensive purulent sputum is due merely to the presence of certain specific infecting organisms, particularly the staphylococcus and bacillus coli.

\section{Chronic Bronchitis}

This condition may present with one of two types of sputum either viscous, jelly-like particles of mucus, purulent to a variable degree (occurring more commonly for example as a result of smoking); or the alternative is a more freely productive cough, with larger quantities of obviously purulent sputum, as is frequently associated with sinusitis.

\section{Bronchiectasis.}

This is a disease mainly of adolescents and young adults, and as the name implies the basic defect is that of dilatation of the bronchi, associated with which there occurs accumulation of secretion with infection, and consequently a productive cough. The underlying cause of the dilatation is probably destruction of muscle and elastic tissue in the wall of the bronchus due to infection during the earlier years of life, e.g. the bronchitis of measles, or that associated with sinusitis, or whooping-cough. In view of the more common incidence in certain portions of the lung (notably the lower lobes, lingula, or middle lobe) a postural factor may be obvious in precipitating
coughing. 
Conservative treatment consists of physiotherapy, with the administration of antibiotics when necessary. But provided that the disease is not so extensive as to make surgical extirpation impossible, this (latter) must remain the treatment of choice in view of the good results obtained, even though bilateral operations may be required to effect a cure.

Before considering resection of any pus-producing lung lesion, it is essential that the infection be controlled as far as possible, and that the secretions be reduced by a prolonged and intensive course of postural drainage, lest complications such as rupture of the bronchus-stump at the site of amputation, or the formation of an abscess in the residual (healthy) lung should occur in the post-operative period.

The "secondary" form of bronchiectasis which follows on some other pre-existing lesion also merits consideration, the underlying conditions being those which lead to accumulation and infection of secretions in a segment or lobe, or atalectasis which does not clear up at an early stage, or fibrosis with resultant distortion of the bronchi. Specific examples are the continued presence of a foreign body or tumour causing partial bronchial obstruction, or a chronic lung abscess, healed or quiescent tuberculosis, fungal infections, or pneumonia due to Friedlander's bacillus. The management of secondary bronchiectasis is identical with that already described.

\section{Lung Abscess.}

Due to the inhalation of infected particles (e.g. dental "tartar", vomitus, or post-nasal discharge) a consolidation occurs in that portion of lung to which the matter gravitates-classically the axillary subsegments of the upper lobe in the case of the patient lying asleep on his side or the apex of the lower lobe in the case of the patient lying on his back, as during anaesthesia for most types of surgery. Subsequently liquefaction and cavitation occur, and eventually rupture of the abscess into the bronchus, causing a productive cough with attendant relief of symptoms in a thus-far severely ill individual.

If adequately treated at this stage (i.e. by means of antibiotics and intensive postural drainage) the majority of cases of acute lung abscess clear up with complete closure of the cavity. However, if drainage through the bronchus is incomplete, thickening of the wall of the cavity due to fibrosis occurs, and early closure thereof is thus prevented, a chronic condition ensuing - that is one which can be cured only by resection of the affected portion of lung. (It is usually accepted that a cavity is unlikely to heal if it has not already done so after two months' therapy).

Drainage may be interfered with by the presence in the cavity of semi-solid material, such as sloughed lung tissue, or the inspirated pus which occurs particularly in cases of Staphylococcal or fungal infection. And furthermore if cavities are multiple with much induration (fibrosis) between them, suggestive again of fungus infection (e.g. actinomycosis), or of preceding Friedlander's pneumonia, little hope can be held out for a complete cure on conservative treatment only.

In children especially, Staphylococcal abscesses may also be multiple and often bilateral, but they differ from the above types in that (in this age-group) the cavities are thin-walled and tend to be large with little reaction surrounding them once they have drained into the bronchi. These cavities are known as pneumatocoeles, and the appearance on X-ray is likened to that of an aggregation of soap-bubbles.

\section{Empyema with Broncho-Pleural Fistula.}

By this is implied the occurrence of pus in the chest (pleural) cavity external to the lung itself, but which drains by means of a communication with the bronchial tree. It very closely mimics a lung abscess, and if loculated and not too large may in fact be treated similarly with a satisfactory result. However, the majority of such cases do require external surgical drainage, although a trial of conservative management is always justified provided that the patient's general condition is not jeopardised thereby.

\section{IN CONCLUSION}

If in discussing the significance of the bronchial secretions a greater understanding of this aspect of "chest physiotherapy" has been brought about, and especially if an increased measure of interest has been instilled into what could be regarded as one of the less pleasant forms of professional application, the writer will be pleased. Needless to say the support of the enthusiastic physiotherapist in the field of thoracic surgery is basically essential for successful practice.

\section{SUMMARY}

1. The effects of accumulated bronchial secretions are enumerated, and physiological and artificial means of eliminating them are discussed.

2. The productive cough. Types of sputum and the significance of each are described, with the accent on suppurative pulmonary conditions and the management thereof.

\section{A. C. MILLER \& CO. ORTHOPAEDIC MECHANICIANS}

Manufacturers and Suppliers of:

ORTHOPAEDIC APPLIANCES, ARTIFICIAL LIMBS, TRUSSES, SURGICAL CORSETS, URINALS, ARCH SUPPORTS, COLOSTOMY BELTS, ELASTIC STOCKINGS, ANKLE GUARDS, WRIST GUARDS, ELBOW GUARDS, KNEE GUARDS, LIGHT DURAL CRUTCHES FOR CHILDREN, WOODEN CRUTCHES, AND METAL ELBOW CRUTCHES.

Phone 23-2496 P.O. Box 3412 312 Bree Street, Johannesburg 